\title{
A Low Aldosterone/Renin Ratio and High Soluble ACE2 Associate with COVID-19 Severity
}

Sakir Akin ( sakirakin@gmail.com )

Erasmus MC https://orcid.org/0000-0003-0700-1930

\section{Paula Schriek}

HagaZiekenhuis Locatie Leyweg: HagaZiekenhuis

\section{Cees van Nieuwkoop}

HagaZiekenhuis Locatie Leyweg: HagaZiekenhuis

\section{Rugina I. Neuman}

Erasmus Medical Centre: Erasmus MC

\section{Iwan Meynaar}

HagaZiekenhuis Locatie Leyweg: HagaZiekenhuis

\section{Erik J. van Helden}

HagaZiekenhuis Locatie Leyweg: HagaZiekenhuis

\section{Hassan El Bouazzaoui}

HagaZiekenhuis Locatie Leyweg: HagaZiekenhuis

\section{Remon Baak}

HagaZiekenhuis Locatie Leyweg: HagaZiekenhuis

Marjan Veuger

HagaZiekenhuis Locatie Leyweg: HagaZiekenhuis

\section{Ronne A.T.A. Mairuhu}

HagaZiekenhuis Locatie Leyweg: HagaZiekenhuis

\section{Lettie van den Berg}

HagaZiekenhuis Locatie Leyweg: HagaZiekenhuis

\section{Vincent van Driel}

HagaZiekenhuis Locatie Leyweg: HagaZiekenhuis

\section{Loes E. Visser}

HagaZiekenhuis Locatie Leyweg: HagaZiekenhuis

\section{Evert de Jonge}

Erasmus Medical Centre: Erasmus MC

\section{Ingrid M. Garrelds}

Erasmus Medical Centre: Erasmus MC

\section{Jan Friso A. B. Duynstee}

HagaZiekenhuis Locatie Leyweg: HagaZiekenhuis Jan Kees van Rooden 
HagaZiekenhuis Locatie Leyweg: HagaZiekenhuis

Jeroen Ludikhuize

Haga Hospital: HagaZiekenhuis

Koen Verdonk

Erasmus Medical Centre: Erasmus MC

\section{Kadir Caliskan}

Erasmus Medical Center: Erasmus MC

\section{Tim Jansen}

HagaZiekenhuis Locatie Leyweg: HagaZiekenhuis

\section{Ron H.N. van Schaik}

Erasmus Medical Centre: Erasmus MC
A.H. Jan Danser
Erasmus Medical Centre: Erasmus MC

\section{Research}

Keywords: ACE2, renin, aldosterone, COVID-19, TMPRSS2

Posted Date: July 24th, 2021

DOl: https://doi.org/10.21203/rs.3.rs-717704/v1

License: (c) (i) This work is licensed under a Creative Commons Attribution 4.0 International License. Read Full License

Version of Record: A version of this preprint was published at Journal of Hypertension on December 1st, 2021. See the published version at https://doi.org/10.1097/HJH.0000000000003054. 


\section{Abstract}

\section{Background}

The severity of COVID-19 after SARS-CoV-2 infection is unpredictable. Angiotensin-converting enzyme-2 (ACE2) is the receptor responsible for coronavirus binding, while subsequent cell entry relies on priming by the serine protease TMPRSS2 (transmembrane protease, serine 2). Although renin-angiotensinaldosterone-system (RAAS) blockers have been suggested to upregulate ACE2, their use in COVID-19 patients is now considered safe. The aim of our study was to investigate parameters that determine COVID-19 severity, focusing on RAAS-components and variation in the genes encoding for ACE2 and TMPRSS2.

\section{Methods}

Adult patients hospitalized due to SARS-CoV-2 infection between May 2020 and October 2020 in the Haga Teaching Hospital were included, and soluble ACE2 (sACE2), renin, aldosterone (in heparin plasma), and polymorphisms in the ACE2 and TMPRSS2 genes (in DNA obtained from EDTA blood) were determined.

\section{Measurements and main results}

Of the 188 patients that were included, 60 were defined as severe COVID-19 (ICU and/or death). These patients more often used antidiabetic drugs, were older, had higher renin and SACE2 levels, lower aldosterone levels, and a lower aldosterone/renin ratio. In addition, they displayed the TMPRSS2rs2070788 AA genotype less frequently. No ACE2 polymorphism-related differences were observed. Multivariate regression analysis revealed independent significance for age, sACE2, the aldosterone/renin ratio, and the TMPRSS2 rs2070788 non-AA genotype as predictors of COVID-19 severity, together yielding a C-index of 0.79 . Findings were independent of the use of RAAS blockers.

\section{Conclusion}

High sACE2, a low aldosterone/renin ration, and having the TMPRSS2 rs2070788 non-AA genotype are novel independent determinants that may help to predict COVID-19 disease severity.

Trial registration: retrospectively registered

\section{Key Messages}

- Severe COVID-19 is characterized by higher renin and SACE2 levels, lower aldosterone levels, a lower aldosterone/renin ratio, and reduced occurrence of the TMPRSS2-rs2070788 AA genotype.

- Determining a cut-off value for the aldosterone/renin ratio that may help to identify COVID-19 patients at risk very early.

- Age is a persisting risk factor and related to severe COVID-19 in all studies. 


\section{Introduction}

The severe acute respiratory syndrome coronavirus-2 (SARS-CoV-2) has resulted in the death of > 4 million people. Male gender, age, thrombotic complications and comorbidities such as hypertension, coronary heart disease, obesity or diabetes associate with an adverse outcome of SARS-CoV-2 infection [1]. A higher incidence and worse outcome has also been reported in ethnic minority groups [2]. Yet, this could still relate to differences in health care disparity, socioeconomic or lifestyle factors. Angiotensinconverting enzyme-2 (ACE2) is the receptor responsible for coronavirus binding, while subsequent cell entry relies on priming by the serine protease TMPRSS2 (transmembrane protease, serine 2). Genetic variation in both ACE2 and TMPRSS2 may be additional determinants of disease severity [3]. Severe COVID-19 patients display elevated soluble ACE2 levels [4], demonstrating that virus infectivity is accompanied by ACE2 shedding. At the same time, serum ACE levels are decreased in COVID-19 patients [5], with the greatest drop occurring in the most severe cases. This parallels the observation that lung injury, as occurring in acute respiratory distress syndrome (ARDS), results in diminished pulmonary ACE activity [6]. A reduction in ACE activity, like treatment with an ACE inhibitor, would be expected to increase renin levels, and to reduce the angiotensin (Ang) II/I ratio in blood. Indeed, higher renin levels have been observed in the more severe cases [7, 8], which coincided with a reduced Ang II/I ratio [8]. However, this concerned small groups of severe patients $(n=12-15)$, and the Ang II/I ratio was compared to that in previous publications. In this study we aimed to identify determinants of COVID-19 severity (mortality and/or ICU admission) in an ethnically diverse population, focusing on components of the reninangiotensin-aldosterone system (RAAS) and genetic variation in the 2 above-mentioned genes.

\section{Materials And Methods}

\section{Study cohort}

This prospective observational study has made use of adult ( $\geq 18$ years of age) COVID-19 patients admitted to the Haga Teaching Hospital, The Hague, Netherlands between May 2020 and October 2020. All patients who were hospitalized with a confirmed SARS-CoV-2 infection and of whom residual blood samples were available, were eligible for participation by opt-out consent. SARS-CoV-2 infection was confirmed on the basis of a positive SARS-CoV-2 viral RNA polymerase-chain-reaction (PCR) test from naso- and oropharyngeal swabs. Residual blood samples ( 2 tubes of heparin blood and 1 tube of EDTA blood) were obtained within 48 hours after hospital/ICU admission. Heparin plasma and whole EDTA blood were stored at $-20^{\circ} \mathrm{C}$ until analysis.

Exclusion occurred in case patients were pregnant or transported to another hospital, if residual blood was absent, or in case of a rejection of previously inclusion by opt-out consent. Patients were classified as having severe COVID-19 (defined as all-cause in-hospital death and/or ICU admission) or non-severe disease (survivors without ICU admission). For each patient, medication prior and during COVID-19, medical history and baseline characteristics, laboratory and microbiological test results, as well as clinical events following hospital and/or ICU admission, were recorded. Co-morbidities included chronic 
heart disease (defined as structural heart disease or arrythmia), chronic hypertension (defined as having a systolic blood pressure of $\geq 140 \mathrm{mmHg}$ and/or diastolic blood pressure $\geq 90 \mathrm{mmHg}$ or the use of antihypertensive medication), diabetes mellitus type I or II, chronic obstructive pulmonary disease (COPD), asthma and chronic kidney disease (defined as an estimated glomerular filtration rate $<30$ $\left.\mathrm{mL} / \mathrm{min} / 1.73 \mathrm{~m}^{2}\right)$.

\section{Biochemical measurements and genotyping}

Plasma renin was measured using a radioimmunometric assay (Cisbio, Saclay, France). Plasma aldosterone was measured by radioimmunoassay (Demeditec, Kiel, Germany). Soluble ACE2 (sACE2) activity was determined making use of an assay based on the synthetic ACE2-specific substrate McaAPK-Dnp (Anaspec, Fremont, USA) originally described by Ye et al. [9]. Measurements were performed in black microtiter plates in a final volume of $100 \mu \mathrm{L}$. Recombinant human ACE2 (rhACE2, range 0.00625$0.5 \mathrm{ng}$; Abcam, Cambridge, UK) or plasma was added to wells containing buffer ( $50 \mathrm{mmol} / \mathrm{L}$ of 4 morpholineethanesulfonic acid, $300 \mathrm{mmol} / \mathrm{L}$ of $\mathrm{NaCl}, 10 \mu \mathrm{mol} / \mathrm{L}$ of $\mathrm{ZnCl}_{2}$, and $0.01 \%$ Triton-X-100, $\mathrm{pH}=$ 6.5), EDTA-free tablets (Roche, Mannheim, Germany) and $10 \mu \mathrm{mol} / \mathrm{L}$ substrate. Blank wells additionally contained $10 \mu \mathrm{mol} / \mathrm{L}$ of the specific ACE2 inhibitor MLN-4760 (Calbiochem, Darmstadt, Germany), added $15 \mathrm{~min}$ before the addition of rhACE2 or plasma. Fluorescence was measured using a microplate fluorescence reader (Gemini EM, Spectramax from Molecular Devices, San Jose, USA) at $320 \mathrm{~nm}$ excitation and $420 \mathrm{~nm}$ emission wavelength. Activity was calculated from the linear fluorescence change between 30-150 min of incubation, and converted to ACE2 concentration based on the activities measured with rhACE2. Levels were corrected for blank values and plasma volume. Routine laboratory measurements were performed in clinical chemistry lab of the Haga Teaching Hospital.

DNA was isolated from fresh frozen EDTA blood with the Maxwell RSC system (Promega Benelux BV) using the whole blood isolation kit (AS1520) according to the manufacturer's protocol. Input volume was set on $400 \mu \mathrm{L}$ and elution volume was $100 \mu \mathrm{L}$. After isolation the DNA was quantified using Nanodrop 1000 and subsequently diluted to $10 \mathrm{ng} / \mu \mathrm{L}$ working solution and stored at $4-7^{\circ} \mathrm{C}$ until analysis. Genotyping assays were supplied by Thermofisher and run on a Taqman 7500 fast system using standard genotyping conditions $\left(1\right.$ minute $60^{\circ} \mathrm{C}, 20$ seconds $95^{\circ} \mathrm{C}, 40$ cycles $95^{\circ} \mathrm{C}$ for 3 seconds and 30 seconds $60^{\circ} \mathrm{C}$ followed by 1 minute $60^{\circ} \mathrm{C}$ post PCR) with $20 \mathrm{ng}$ DNA input. A control sample and blanks were included in every run. We included ACE2 rs12551879 (T > C), a SNP representative for the COVID-19 binding region, and TMPRSS2 rs2070788 (A > G) and rs8134378 ( $\mathrm{G}>\mathrm{A})$, SNPs that are involved in TPRMSS2 expression.

\section{Statistical analysis}

Baseline characteristics between non-severe and severe patients were described using mean ( \pm SD) or median (interquartile range) in the case of non-parametric distributions, and number (percentage) for categorical variables. The Shapiro-Wilk $W$ test was applied to assess the normality of continuous variables. For the comparison of continuous data between 2 groups, unpaired Student's t-test or Mann Whitney U for non-normally distributed data was applied. Fisher's exact and $\mathrm{X}^{2}$ (Chi-square) were 
performed to evaluate differences between categorical variables. To investigate correlations between SACE2, renin and aldosterone in severe and non-severe patients, the Pearson correlation coefficient was calculated.

To study the association between the dichotomous outcomes (severe vs. non-severe disease) and candidate predictors, logistic regression analysis was performed. Traditional risk factors and RAAS components with a P-value of $<0.10$ between the severe and non-severe group (i.e., age, diabetes, sACE2, renin, aldosterone, aldosterone/renin ratio, and TMPRSS2 rs2070788 AA genotype) were analyzed as candidate predictors. Missing data on these predictors were imputed using multivariate imputation by chained equations. The relation between each predictor variable and the outcome (severe disease) was assessed by univariable logistic regression. Non-linearity in associations between continuous candidate predictors and the risk of developing severe disease was assessed using logarithm transformations.

Furthermore, we sought to identify which set of candidate predictors would best estimate the risk of severe disease (ICU admission and/or all-cause death). Due to the high correlation between renin, aldosterone and the aldosterone/renin ratio, only the aldosterone/renin ratio was taken into account (indeed a model with aldosterone/renin ratio rather than aldosterone or renin alone gave the best fit). Subsequently, a backward selection procedure was applied to limit the number of predictors in the final model. Model specification was based on the Akaike Information Criterion (AIC) in a backward selection procedure, which is equivalent to the exclusion of candidate predictors when $P>0.157$. To assess the discriminative ability of the model we used the $\mathrm{C}$-index, which is equivalent to the area under the ROC curve for dichotomous outcomes. Discrimination is defined as how well a model distinguishes between severe and non-severe infection. The c-statistic ranges from 0.5 for a model equivalent to a coin toss to 1.0 for a model with perfect discrimination. SPSS Statistics 21 (IBM Corporations) and R Software were used for the statistical analysis.

\section{Results}

\section{Study cohort}

Of the 240 adult COVID-19 patients included in our database of whom residual blood samples were available between May 2020 and October 2020, 52 were excluded because of missing residual blood ( $n=$ 2), rejection of previous inclusion by opt-out consent $(n=8)$, or other exclusion criteria such as pregnancy, negative PCR and transportation to another hospital $(n=42)$ (Fig. 1). This resulted in a final study population of 188 patients with a mean age of 62 years and a mean BMl of $29 \mathrm{~kg} / \mathrm{m}^{2}$, of whom the majority $(70 \%)$ was male (Table 1$)$. 
Table 1

Baseline and demographic parameters in severe and non-severe COVID-19 patients.

\begin{tabular}{|c|c|c|c|c|}
\hline & $\begin{array}{l}\text { Total } \\
(n=188)\end{array}$ & $\begin{array}{l}\text { Non-Severe }(n \\
=128)\end{array}$ & $\begin{array}{l}\text { Severe } \\
(n=60)\end{array}$ & $\begin{array}{l}\mathrm{P} \text { - } \\
\text { value }\end{array}$ \\
\hline \multicolumn{5}{|l|}{ Gender, n (\%) } \\
\hline Male & 132 & $90(70.3)$ & $42(70.0)$ & 0.97 \\
\hline Female & 56 & $38(29.7)$ & $18(30.0)$ & \\
\hline Age, years & $61.5(15.9)$ & $59.0(15.3)$ & $66.9(15.9)$ & 0.01 \\
\hline Length, cm ( $n=71 / 47)$ & $172.5(9.7)$ & $172.4(9.3)$ & $172.7(10.3)$ & 0.90 \\
\hline Weight, kg (n= 87/48) & $86.6(21.3)$ & $87.3(20.7)$ & $85.2(22.7)$ & 0.59 \\
\hline $\mathrm{BMI}, \mathrm{kg} / \mathrm{m}^{2}(\mathrm{n}=65 / 44)$ & $29.2(6.7)$ & $29.3(6.1)$ & $29.2(7.5)$ & 0.93 \\
\hline Ethnic Background, n (\%) & & & & 0.28 \\
\hline Caucasian/White & 77 & $48(37.5)$ & $29(48.3)$ & 0.16 \\
\hline African/Black & 7 & $4(3.1)$ & $3(5.0)$ & 0.68 \\
\hline Turkish/Moroccan & 55 & $42(32.8)$ & $13(21.7)$ & 0.12 \\
\hline Surinamese/Antillean & 34 & $21(16.4)$ & $13(21.7)$ & 0.38 \\
\hline Other & 15 & $13(10.1)$ & $2(3.3)$ & 0.15 \\
\hline \multicolumn{5}{|l|}{ Clinical manifestations at admission } \\
\hline Temperature, ${ }^{\circ} \mathrm{C}$ & $38.1(0.9)$ & $38.1(0.9)$ & $38.1(1.0)$ & 0.54 \\
\hline Respiratory Rate, $\mathrm{n} / \mathrm{min}$ & $25.6(7.0)$ & $24.6(6.0)$ & $28.0(8.4)$ & 0.002 \\
\hline SBP, mm Hg & $140(24)$ & $140(24)$ & $139(25)$ & 0.73 \\
\hline $\mathrm{DBP}, \mathrm{mm} \mathrm{Hg}$ & $80(17)$ & $82(17)$ & $78(17)$ & 0.15 \\
\hline Oxygen Saturation, \% & $93.6(4.4)$ & $94.3(3.2)$ & $92.1(6.1)$ & 0.002 \\
\hline $\begin{array}{l}\text { Arterial Partial Pressure of Oxygen, } \mathrm{kPa} \\
(\mathrm{n}=113 / 55)\end{array}$ & $\begin{array}{l}8.9(8.0- \\
9.9)\end{array}$ & $8.8(8.0-9.8)$ & $9.2(7.8-11)$ & 0.24 \\
\hline Oxygen supplementation, n (\%) & 83 & $52(40.6)$ & $31(52.5)$ & 0.24 \\
\hline \multicolumn{5}{|l|}{ Laboratory parameters at admission } \\
\hline Hemoglobin, $g / L$ & $8.3(1.2)$ & $8.4(1.1)$ & $8.2(1.3)$ & 0.41 \\
\hline Hematocrit, \% & $0.39(0.05)$ & $0.40(0.05)$ & $0.39(0.06)$ & 0.41 \\
\hline White blood cells, $x 10^{9} / \mathrm{L}$ & $7.0(5.0-9.2)$ & $6.9(4.7-8.7)$ & $\begin{array}{l}7.5(5.4- \\
10.9)\end{array}$ & 0.06 \\
\hline
\end{tabular}




\begin{tabular}{|c|c|c|c|c|}
\hline & $\begin{array}{l}\text { Total } \\
(n=188)\end{array}$ & $\begin{array}{l}\text { Non-Severe ( } \mathrm{n} \\
=128 \text { ) }\end{array}$ & $\begin{array}{l}\text { Severe } \\
(n=60)\end{array}$ & $\begin{array}{l}P \text { - } \\
\text { value }\end{array}$ \\
\hline Lymphocytes, $x 10^{9} / L(n=121 / 40)$ & $\begin{array}{l}1.0(0.72- \\
1.4)\end{array}$ & $1.1(0.75-1.5)$ & $\begin{array}{l}0.87(0.68- \\
1.1)\end{array}$ & 0.03 \\
\hline Neutrophils, $x 10^{9} / L(n=121 / 40)$ & $\begin{array}{l}4.9(3.2- \\
6.8)\end{array}$ & $4.8(3.1-6.6)$ & $\begin{array}{l}5.3(3.4- \\
7.3)\end{array}$ & 0.18 \\
\hline Ferritin, $\mu \mathrm{g} / \mathrm{L}(\mathrm{n}=115 / 37)$ & $\begin{array}{l}590(286- \\
1237)\end{array}$ & $\begin{array}{l}592(273- \\
1228)\end{array}$ & $\begin{array}{l}552(326- \\
1325)\end{array}$ & 0.50 \\
\hline $\mathrm{ALT}, \mathrm{U} / \mathrm{L}(\mathrm{n}=127 / 52)$ & $31(21-48)$ & $32(22-46)$ & $31(21-56)$ & 0.75 \\
\hline AST, U/L $(n=120 / 47)$ & $45(32-62)$ & $42(31-60)$ & $52(40-75)$ & 0.02 \\
\hline Creatinine, $\mu \mathrm{mol} / \mathrm{L}$ & $80(63-100)$ & $77(65-98)$ & $90(60-114)$ & 0.20 \\
\hline Creatine Kinase, U/L & $\begin{array}{l}135(80- \\
228)\end{array}$ & $135(82-198)$ & $\begin{array}{l}144(65- \\
315)\end{array}$ & 0.71 \\
\hline $\mathrm{LDH}, \mathrm{U} / \mathrm{L}(\mathrm{n}=118 / 53)$ & $\begin{array}{l}347(262- \\
449)\end{array}$ & $328(252-405)$ & $\begin{array}{l}429(311- \\
543)\end{array}$ & 0.000 \\
\hline Sodium, $\mathrm{mmol} / \mathrm{L}$ & $135(4.3)$ & $134(4.1)$ & $135(4.7)$ & 0.15 \\
\hline Potassium, mmol/L ( $n=126 / 57)$ & $3.9(0.46)$ & $3.9(0.41)$ & $4.0(0.54)$ & 0.15 \\
\hline D-dimer, mg/L ( $n=119 / 35)$ & $\begin{array}{l}0.35(0.24- \\
0.71)\end{array}$ & $\begin{array}{l}0.31(0.22- \\
0.54)\end{array}$ & $\begin{array}{l}0.72(0.31- \\
1.55)\end{array}$ & 0.000 \\
\hline Platelet count, $\times 10^{9} / \mathrm{L}$ & $\begin{array}{l}210(162- \\
267)\end{array}$ & $207(63-273)$ & $\begin{array}{l}211(159- \\
256)\end{array}$ & 0.62 \\
\hline NT-proBNP, pmol/L $(n=113 / 38)$ & $\begin{array}{l}103(34- \\
422)\end{array}$ & $88(28.5-219)$ & $\begin{array}{l}238(62- \\
1206)\end{array}$ & 0.001 \\
\hline C-reactive Protein, $\mathrm{mg} / \mathrm{L}$ & $74(41-134)$ & $67(35-109)$ & $\begin{array}{l}102(52- \\
196)\end{array}$ & 0.001 \\
\hline sACE2, ng/mL $(n=124 / 59)$ & $\begin{array}{l}0.50(0.50- \\
4.2)\end{array}$ & $0.50(0.50-1.2)$ & $\begin{array}{l}2.47(0.50- \\
9.3)\end{array}$ & 0.000 \\
\hline Renin, $\mathrm{pg} / \mathrm{mL}(\mathrm{n}=124 / 59)$ & $\begin{array}{l}28.8(12.3- \\
59.7)\end{array}$ & $\begin{array}{l}23.1(11.6- \\
45.6)\end{array}$ & $\begin{array}{l}42.7(14.4- \\
67.1)\end{array}$ & 0.02 \\
\hline Aldosterone, $\mathrm{pg} / \mathrm{mL}(\mathrm{n}=124 / 59)$ & $\begin{array}{l}127(75- \\
233)\end{array}$ & $137(87-238)$ & $\begin{array}{l}107(41- \\
213)\end{array}$ & 0.003 \\
\hline Aldosterone/Renin Ratio $(n=124 / 59)$ & $\begin{array}{l}5.9(2.2- \\
11.3)\end{array}$ & $7.6(3.7-12.6)$ & $\begin{array}{l}2.3(0.8- \\
9.0)\end{array}$ & 0.000 \\
\hline $\begin{array}{l}\text { Cycle time (Ct)-values of PCR }(n= \\
76 / 48)\end{array}$ & $\begin{array}{l}24.6(20.9- \\
28.3)\end{array}$ & $\begin{array}{l}25.1(21.3- \\
28.0)\end{array}$ & $\begin{array}{l}23.3(19.4- \\
29.0)\end{array}$ & 0.56 \\
\hline
\end{tabular}




\begin{tabular}{|c|c|c|c|c|}
\hline & $\begin{array}{l}\text { Total } \\
(n=188)\end{array}$ & $\begin{array}{l}\text { Non-Severe (n } \\
=128)\end{array}$ & $\begin{array}{l}\text { Severe } \\
(n=60)\end{array}$ & $\begin{array}{l}\mathrm{P} \text { - } \\
\text { value }\end{array}$ \\
\hline Chronic Heart Disease & 39 & $23(18.1)$ & $16(26.7)$ & 0.18 \\
\hline Hypertension & 63 & $38(29.7)$ & $25(41.7)$ & 0.11 \\
\hline Diabetes Mellitus & 58 & $33(25.8)$ & $25(41.8)$ & 0.06 \\
\hline COPD & 21 & $14(10.9)$ & $7(11.7)$ & 0.88 \\
\hline Asthma & 17 & $13(10.2)$ & $4(6.7)$ & 0.64 \\
\hline Chronic Kidney Disease & 16 & $9(7.0)$ & $7(11.7)$ & 0.46 \\
\hline \multicolumn{5}{|l|}{ Medication Use, n (\%) } \\
\hline Steroid & 15 & $8(6)$ & $7(12)$ & 0.20 \\
\hline Diuretic & 33 & $22(17)$ & $11(18)$ & 0.85 \\
\hline ACE inhibitor & 25 & $14(10.9)$ & $11(18.3)$ & 0.16 \\
\hline ARB & 26 & $17(13.3)$ & $9(15)$ & 0.75 \\
\hline Metformin & 46 & $26(20.3)$ & $20(33.3)$ & 0.05 \\
\hline Other antidiabetic drugs & 17 & $8(6.3)$ & $9(15)$ & 0.05 \\
\hline Insulin & 18 & $8(6.3)$ & $10(16.7)$ & 0.02 \\
\hline \multicolumn{5}{|l|}{ Polymorphisms } \\
\hline \multicolumn{5}{|c|}{ TMPRSS2 rs2070788 (n= 114/55) } \\
\hline AA & 56 & $43(37.7 \%)$ & $13(23.6 \%)$ & \\
\hline$A G / G G$ & 113 & $71(62.3 \%)$ & $42(76.4 \%)$ & 0.07 \\
\hline \multicolumn{5}{|c|}{ TMPRSS2 rs8134378 ( $n=113 / 54)$} \\
\hline GG & 130 & $84(74.3 \%)$ & $46(85.2 \%)$ & \\
\hline $\mathrm{AA} / \mathrm{AG}$ & 37 & $29(25.7 \%)$ & $8(14.8 \%)$ & 0.11 \\
\hline \multicolumn{5}{|c|}{ ACE2 rs12551879 $(n=114 / 55)$} \\
\hline TT & 142 & $98(86.0 \%)$ & $44(80.0 \%)$ & \\
\hline СT/CC & 27 & $16(14.0 \%)$ & $11(20.0 \%)$ & 0.32 \\
\hline
\end{tabular}




$\begin{array}{llll}\text { Total } & \text { Non-Severe }(n & \text { Severe } & \begin{array}{l}\text { P- } \\ \text { value }\end{array} \\ (n=188) & & (n=60) & \end{array}$

Categorical variables are presented as frequencies and percentages. Continuous variables are presented as mean (standard deviation) or median (interquartile range). In case parameters were not determined in all patients, the correct numbers are provided in the left column. SACE2, soluble angiotensin-converting enzyme 2; ALT, alanine-aminotransferase; ARB, angiotensin II type 1 receptor blocker; AST, aspartate transaminase; COPD, chronic obstructive pulmonary disease; COVID-19, coronavirus disease 2019; LDH, lactate dehydrogenase; SBP, systolic blood pressure; DBP, diastolic blood pressure; PCR, polymerase chain reaction; NT-proBNP, N-terminal prohormone brain natriuretic peptide; TMPRSS2, transmembrane protease, serine 2; A, adenine; C, cytosine; G, guanine; and T, thymine. The statistical differences between the severe and non-severe COVID-19 were calculated using the non-parametric Mann Whitney U test (for continuous variables), chi-square and Fisher's exact test (for categorical variables).

\section{Severe versus non-severe COVID-19}

The main reason for ICU admission was respiratory failure due to acute respiratory distress syndrome, characterized by a higher respiratory rate and lower oxygen saturation (Table 1). Death occurred in 27 patients with severe COVID-19. Diabetes tended to occur more frequently among ICU patients, as did the use of metformin, insulin, and antidiabetic drugs other than metformin and insulin ( $P=0.05$ for all). Severe patients displayed increased levels of lactate dehydrogenase, C-reactive protein, $\mathrm{N}$-terminal prohormone brain natriuretic peptide, and D-dimers. Their aldosterone levels were lower, and their renin levels were higher. As a consequence, their aldosterone/renin ratio was greatly reduced as compared to the non-severe group.

These results were unaltered when excluding patients using RAAS inhibitors (data not shown). Eight patients received intravenous furosemide during admission, of which 2 were already being treated orally with a diuretic before hospital admission. Four of these 8 patients had severe COVID-19 disease. Of the 8 patients receiving dexamethasone during admission, five had severe COVID-19 disease. Additionally, seven patients were already being treated with a steroid before hospital admission, of which two had severe disease. Table 1 shows that the use of either diuretics or steroids (at home and/or acutely during admission) was identical in both groups, and consequently correcting for the use of diuretics or steroids also did not alter the results (data not shown).

The TMPRSS2 rs2070788 AA genotype tended to occur less frequently in ICU patients $(P=0.07)$. None of the other SNPs from either the TMPRSS2 gene or the ACE2 gene displayed significant differences.

\section{Prediction of severe COVID-19}

We performed univariate logistic regression with traditional risk factors (age and diabetes) and RAAS components displaying a P-value $<0.10$. The aldosterone/renin ratio was used instead of renin and aldosterone separately. Diabetes was used rather than the use of antidiabetic drugs. This approach confirmed that all parameters that were significant at $\mathrm{P}<0.10$ in Table 1 could predict severe COVID-19 with C-indexes ranging from $0.58-0.68$ (Table 2). The effect of both sACE2 and the aldosterone/renin ratio was unaltered after correcting for hypertension or the use of RAAS inhibitors, diuretics or steroids 
(data not shown). Finally, incorporating all parameters in a multivariate regression analysis revealed independent significance for age, sACE2, the aldosterone/renin ratio, and the TMPRSS2 rs2070788 AA genotype, together yielding a C-index of 0.79 .

Table 2

Univariate regression of parameters that predict severe COVID-19.

\begin{tabular}{llll}
\hline Parameter & OR $(95 \% \mathrm{Cl})$ & C-index & P \\
\hline Traditional predictors & & & \\
\hline Age ${ }^{\star}$, years & $2.01(1.30-3.12)$ & 0.64 & 0.0017 \\
\hline Diabetes Mellitus & $2.06(1.08-3.93)$ & 0.58 & 0.0293
\end{tabular}

\section{RAAS components}

SACE2, $\mathrm{ng} / \mathrm{mL}$

$1.42(1.21-1.67)$

0.68

$<0.0001$

Renin, $\mathrm{pg} / \mathrm{mL}$

$1.23(1.01-1.51)$

0.61

0.04

Aldosterone, $\mathrm{pg} / \mathrm{mL}$

$0.61(0.47-0.79)$

0.64

$<0.0001$

Aldosterone/Renin ratio

$0.69(0.57-0.84)$

0.68

$<0.0001$

\section{Polymorphism}

TMPRSS2 rs2070788 AA genotype

$0.44(0.21-0.91)$

0.59

0.04

Continuous variables were logarithmically transformed (log2). The odds ratio and its associated $95 \%$ $\mathrm{Cl}$ can be interpreted as the odds of developing severe disease when the continuous variable multiplies by 2. *Interquartile odds ratio and associated $95 \%$ confidence interval was calculated to aid interpretation of the continuous variable age. It is defined as comparing the risk of severe disease at the 75th percentile of the marker value versus the 25th percentile. SACE2, soluble angiotensinconverting enzyme-2; RAAS, renin-angiotensin-aldosterone system; TMPRSS2, transmembrane protease, serine 2 .

Table 3

Multivariate regression analysis of factors that predict severe COVID-19.

\begin{tabular}{llll} 
Parameter & OR $(95 \% \mathrm{Cl})$ & C-index & P \\
\cline { 1 - 2 } Age $^{\star}$ years & $2.01(1.23-3.28)$ & 0.79 & 0.004 \\
\cline { 1 - 2 }, $\mathrm{ng} / \mathrm{mL}$ & $1.33(1.11-1.59)$ & & 0.001 \\
Aldosterone/Renin ratio & $0.73(0.59-0.89)$ & & 0.0037 \\
TMPRSS2 rs2070788 AA genotype & $0.37(0.16-0.87)$ & & 0.03
\end{tabular}

Continuous variables were logarithmically transformed (log2). The odds ratio and its associated $95 \%$ $\mathrm{Cl}$ can be interpreted as the odds of developing severe disease when the continuous variable multiplies by 2 . *Interquartile odds ratio and associated $95 \%$ confidence interval was calculated to aid interpretation of the continuous variable age. It is defined as comparing the risk of severe disease at the 75th percentile of the marker value versus the 25th percentile. SACE2, soluble angiotensinconverting enzyme-2; TMPRSS2, transmembrane protease, serine 2. 
Renin did not correlate with SACE2, neither in subjects taking RAAS inhibitors, nor in subjects not taking these drugs (Fig. 2). As expected, in non-severe COVID-19 patients renin correlated with aldosterone in those not taking RAAS inhibitors, but not in those taking such drugs (Figs. 3A and 3C). Unexpectedly, renin did not correlate with aldosterone in severe COVID-19 patients taking or not taking RAAS inhibitors (Figs. 3B and 3D).

\section{Discussion}

This study is the first to describe that COVID-19 severity coincides with a reduced aldosterone/renin ratio in an ethnically diverse population. This occurred independently of the use of ACEi/ARBs, diuretics or steroids. Simultaneously we confirmed that severe COVID-19 patients were older, had more diabetes and displayed elevated SACE2 levels, although after multivariate regression analysis of these 3 parameters only age and SACE2 remained as independent determinants of severity. We also observed an independent protective role for the TMPRSS2 rs2070788 AA genotype as a determinant of severity, which likely reflects the role of this protease in coronavirus entry, given that this genotype corresponds with low TMPRSS2 expression [10]. Yet, we were unable to demonstrate associations with a SNP in the COVID-19-binding region of ACE2.

In non-severe COVID-19 patients not taking RAAS inhibitors, renin correlated with aldosterone. This relationship reflects the fact that Ang II stimulates the synthesis and release of aldosterone [11]. Not surprisingly, such a relationship was absent in non-severe COVID-19 patients taking RAAS inhibitors, as well as in severe COVID-19 patients taking these drugs. Yet, unexpectedly there also was no significant relationship between renin and aldosterone in severe COVID-19 patients not taking RAAS inhibitors. Thus, these patients resemble those on ACEi/ARBs, although they did not take these drugs. A logical explanation would be the previous observation that COVID-19 patients display reduced ACE levels [5]. This is due to the fact that severe lung injury (as occurring in severe COVID-19 patients) induces endothelial dysfunction and loss of pulmonary ACE expression [6]. Here it is important to note that the majority of ACE is expressed on the surface of endothelial cells [12]. Circulating ACE is derived from ACEexpressing cells by proteolytic cleavage at the juxtamembrane stalk region [13]. Given its rich expression of ACE, the lung is the most important source of circulating ACE in the body, which makes a drop in circulating ACE in severe COVID-19 patients with lung injury likely. A reduction in ACE, via diminished Ang II formation, would suppress aldosterone, and upregulate renin, in an attempt to restore Ang II. As a consequence, the renin-aldosterone relationship is disturbed, and the aldosterone/renin ratio is lowered.

Simultaneously, we observed elevated levels of sACE2 in severe COVID-19 patients, in agreement with earlier observations [4]. This occurred fully independently of the aldosterone/renin ratio. In support of our findings, incubating serum samples of severe COVID-19 patients for 1 hour, resulted in increased formation of Ang-(1-7) when compared with non-severe patients [4]. Since ACE2 generates Ang-(1-7) from Ang II, this observation nicely reflects the increased levels of sACE2 in serum. However, to what degree such ex-vivo observations imply that the circulating Ang-(1-7) levels in severe COVID-19 patients are elevated as well cannot be concluded from these data, since normally angiotensin generation and 
metabolism occurs by membrane-bound angiotensinases, and not by the small percentage of these enzymes (including ACE and ACE2) that occurs in a soluble form in blood [14]. One might argue that SACE2 contributes to Ang II degradation. If so, patients with high sACE2 levels should have high renin levels to match the increased Ang II degradation. As shown in Fig. 2, our data do not support this concept. Consequently, sACE2 does not appear to be a major determinant of Ang II metabolism in vivo, as has been suggested before [15]. Importantly, ACE2 expression in the lungs is low, as compared to kidney and gastrointestinal tract [16]. Circulating ACE2, unlike ACE, is therefore unlikely to be lung-derived. This may simultaneously explain why the alterations in SACE2 and the aldosterone/renin ratio (as a reflection of reduced ACE activity) are both independent determinants of COVID-19 severity. Whether the increase in SACE2 is due to increased ACE2 shedding (potentially resulting in diminished local ACE2 activity), a general ACE2 upregulation in severe COVID-19, or both, cannot yet be said, and would require a detailed insight in the tissue expression of ACE2.

Attempts have been made to measure Ang II levels in COVID-19 patients and controls. This usually concerned small patient groups [8,17-19], and in all cases the levels were many orders of magnitude above those reported in humans before $[11,20,21]$. Hence, this seriously hampers the interpretation of these data, as it points to either inappropriate collection of blood samples (i.e., without inhibitors of angiotensinases), the use of unreliable assays [22] or both. Yet, with one exception [17], no increased levels of Ang II were reported in COVID-19 patients in any of these studies. Others incubated serum of COVID-19 patients $[4,23,24]$ and observed diminished, unaltered as well as increased Ang II generation (defined as the Ang II level measured after one hour of incubation ay $37^{\circ} \mathrm{C}$ ). This outcome may relate to the severity of COVID-19, the concomitant use of ACEi/ARBs, and the occurrence of comorbidities (hypertension and heart failure) in both controls and COVID-19 patients. Moreover, ex-vivo Ang II generation not necessarily reflects in-vivo Ang II levels. Thus, at present, no clear picture can be provided of the Ang II levels in COVID-19 patients.

At the start of the COVID-19 pandemic, it has been suggested that RAAS inhibitors would increase the susceptibility for COVID-19, given that such drugs were believed to upregulate ACE2 and/or affect ACE2 shedding $[3,25]$. Overwhelming data currently argue against this concept,[26-28] if not supporting the opposite [29]. In fact, a recent systematic review concluded that ACE2 overexpression is a rare rather than common consequence of RAAS inhibitor treatment in both healthy animals and disease models [30]. Our findings, illustrating that severe COVID-19 patients may already be in a state of RAAS inhibition, independently of the use of ACEi/ARBs, provide additional evidence why it is unlikely that such an association exists. Hence, combining the measurement of the aldosterone/renin ratio with the determination of the TMPRSS2 rs2070788 genotype may actually help to identify severe COVID-19 patients. Currently, the aldosterone/renin ratio is already used to identify patients with primary hyperaldosteronism, in whom this ratio is increased [31].

\section{Strengths of this study}


By determining RAAS parameters and genetic variation in the gene encoding for TMPRSS2 in 188 patients, we observed that severe COVID-19 (ICU and/or death) is characterized by higher renin and SACE2 levels, lower aldosterone levels, a lower aldosterone/renin ratio, and reduced occurrence of the TMPRSS2-rs2070788 AA genotype. In combination with age these parameters yielded a C-index of 0.79 to predict disease severity. The fact that COVID-19 is characterized by a low aldosterone/renin ratio suggests that the disease itself results in a situation that mimics RAAS blockade. A likely cause is reduced pulmonary ACE due to lung injury in COVID-19 patients. Determining a cut-off value for the aldosterone/renin ratio may help to identify COVID-19 patients at risk.

\section{Limitations}

A limitation of our study is the fact that we neither measured ACE nor angiotensins. Indeed, ideally, we would have verified the reduction in pulmonary ACE activity demonstrated earlier with elegant in-vivo techniques in ARDS patients [6]. Angiotensin measurement would have required the careful collection of blood samples with angiotensinase inhibitors, combined with an established assay excluding the detection of significant background signals [22]. Finally, we should now determine a cut-off value for the aldosterone/renin ratio that may help to identify COVID-19 patients at risk. This will require larger patient numbers, and preferably a prospective clinical trial evaluating this value, analogous to the trials that have been performed to establish this ratio in primary hyperaldosteronism.

\section{Conclusions}

Using antidiabetic drugs, older age, higher renin and SACE2 levels, lower aldosterone levels, and a lower aldosterone/renin ratio at admission are associated with ICU admission and/or death after SARS-CoV-2 infection. High SACE2, a low aldosterone/renin ratio, and having the TMPRSS2 rs2070788 non-AA genotype are novel independent determinants that may help to predict COVID-19 disease severity.

\section{Abbreviations}

ACE-2

Angiotensin-converting enzyme-2

ARB

Angiotensin II type 1 receptor blocker

ARDS

Acute respiratory distress syndrome

COVID-19

Coronavirus disease 2019

RAAS

Renin-angiotensin-aldosterone system

\section{SARS-CoV-2}

Severe acute respiratory syndrome coronavirus type 2

TMPRSS2 


\section{Declarations}

* Ethics approval and consent to participate: The ethics review board of The Hague Hospital (number T20-054) approved the study protocol, and written informed consent was obtained from all participants after enrollment. This prospective observational study has made use of data as part of the International Severe Acute Respiratory and Emerging Infection Consortium (ISARIC). All patients who were hospitalized with a confirmed SARS-CoV-2 infection and of whom residual blood samples were available, were eligible for participation by opt-out consent.

* Consent for publication: Exclusion occurred in case patients were pregnant or transported to another hospital, if residual blood was absent, or in case of a rejection of previously inclusion by opt-out consent.

* Availability of data and materials: The datasets used and/or analyzed during the current study are available from the corresponding author on reasonable request.

* Competing interests: Not applicable

* Funding: This work was supported by Haga Science Department in the Hague, The Netherlands

* Authors' contributions: SA, PS, CvN, RN, RvS and JD conceived the study protocol; SA, PS, CvN, RN, IM, EvH, HelB, RB, MV, RM, LvdB, VvD, LV, EdeJ, IG, JFD, JKvR, JL, KV, KC, TJ, RvS and JD participated in the design and coordination of the study; SA, PS, CvN, RN, EvH, RvS and JD collected study data; SA, PS, CvN, $\mathrm{RN}, \mathrm{IM}, \mathrm{EvH}, \mathrm{KV}, \mathrm{RvS}$ and JD participated in data interpretation; SA, PS, CvN, RN, IM, EvH, HelB, RB, MV, RM, VvD, LV, EdeJ, IG, KV, RvS and JD drafted the present manuscript; SA, PS, CvN, RN, IM, EvH, HelB, RB, $M V, R M, V v D, L V$, EdeJ, IG, KV, RvS and JD revised the manuscript. All authors read and approved the final version of the manuscript.

* Acknowledgements: We would like to thank all members of the Haga Teaching Hospital (HagaZiekenhuis) in The Hague, including those helping with setting up the database. We specially acknowledge A. Khalifa, S. Susan and B. Harjo (data collection of the ICU patients), N. van Burgel and I. Pollard (Department of Medical Microbiology), R. Rombley, S. Nieburg and M. Slinger (Clinical Chemistry Laboratory), all ICU nurses, and the intensivists M. Koeman, I. Purmer, T. Ottens, S. Grewal and R. Nowitzky.

We thank the Haga Science Department for their financial support.

* Authors' information (optional): Sakir Akin1,2, Paula Schriek3, Cees van Nieuwkoop4, Rugina I. Neuman5, Iwan Meynaar1, Erik J. van Helden4, Hassan El Bouazzaoui3, Remon Baak1, Marjan Veuger6, Ronne A.T.A. Mairuhu4, Lettie van den Berg1, Vincent van Driel7, Loes E. Visser8, Evert de Jonge9, Ingrid M. Garrelds5, Jan Friso A. B. Duynstee10, Jan Kees van Rooden11, Jeroen Ludikhuize1, Koen Verdonk5, Kadir Caliskan2, Tim Jansen1, Ron H.N. van Schaik9, and A.H. Jan Danser5. 
1Department of Intensive Care, Haga Teaching Hospital, The Hague, The Netherlands;

2Department of Cardiology, Unit Heart Failure and Transplant Unit, Erasmus MC University Medical Center, Rotterdam, The Netherlands;

3Department of Pulmonology, Haga Teaching Hospital, The Hague, The Netherlands;

4Department of Internal Medicine, Haga Teaching Hospital, The Hague, The Netherlands;

5Department of Internal Medicine, Division of Vascular Medicine and Pharmacology Erasmus MC University Medical Center, Rotterdam, The Netherlands;

6Department of Clinical Chemistry, Haga Teaching Hospital, The Hague, The Netherlands;

7Department of Cardiology, Haga Teaching Hospital, The Hague, The Netherlands;

8Department of Hospital Pharmacy, Haga Teaching Hospital, The Hague, The Netherlands;

9Department of Clinical Chemistry, Erasmus MC University Medical Center, Rotterdam, The Netherlands;

10Department of Haga Science, Haga Teaching Hospital, The Hague, The Netherlands;

11Department of Radiology, Haga Teaching Hospital, The Hague, The Netherlands.

\section{Corresponding authors}

Sakir Akin, MD, PhD

\section{E-mail: s.akin@hagaziekenhuis.nl}

\section{References}

1. Guan WJ, Ni ZY, Hu Y, Liang WH, Ou CQ, He JX, Liu L, Shan H, Lei CL, Hui DSC, et al. Clinical characteristics of coronavirus disease 2019 in China. N Engl J Med. 2020;382(18):1708-20.

2. Alcendor DJ. Racial disparities-associated COVID-19 mortality among minority populations in the US. J Clin Med. 2020;9(8):2442.

3. Danser AHJ, Epstein M, Batlle D. Renin-angiotensin system blockers and the COVID-19 pandemic: at present there is no evidence to abandon renin-angiotensin system blockers. Hypertension. 2020;75:1382-5.

4. Reindl-Schwaighofer R, Hodlmoser S, Eskandary F, Poglitsch M, Bonderman D, Strassl R, Aberle JH, Oberbauer R, Zoufaly A, Hecking M: Angiotensin-converting enzyme 2 (ACE2) elevation in severe COVID-19. Am J Respir Crit Care Med 2021:in press.

5. Zhu Z, Cai T, Fan L, Lou K, Hua X, Huang Z, Gao G. The potential role of serum angiotensinconverting enzyme in coronavirus disease 2019. BMC Infect Dis. 2020;20(1):883. 
6. Orfanos SE, Armaganidis A, Glynos C, Psevdi E, Kaltsas P, Sarafidou P, Catravas JD, Dafni UG, Langleben $D$, Roussos $C$. Pulmonary capillary endothelium-bound angiotensin-converting enzyme activity in acute lung injury. Circulation. 2000;102(16):2011-8.

7. Villard O, Morquin D, Molinari N, Raingeard I, Nagot N, Cristol JP, Jung B, Roubille C, Foulongne V, Fesler P, et al. The plasmatic aldosterone and C-reactive protein levels, and the severity of Covid-19: the Dyhor-19 study. J Clin Med. 2020;9(7):2315.

8. Eleuteri D, Montini L, Cutuli SL, Rossi C, Alcaro F, Antonelli M. Renin-angiotensin system dysregulation in critically ill patients with acute respiratory distress syndrome due to COVID-19: a preliminary report. Crit Care. 2021;25(1):91.

9. Ye M, Wysocki J, Gonzalez-Pacheco FR, Salem M, Evora K, Garcia-Halpin L, Poglitsch M, Schuster M, Batlle D. Murine recombinant angiotensin-converting enzyme 2: effect on angiotensin II-dependent hypertension and distinctive angiotensin-converting enzyme 2 inhibitor characteristics on rodent and human angiotensin-converting enzyme 2. Hypertension. 2012;60(3):730-40.

10. Cheng Z, Zhou J, To KK, Chu H, Li C, Wang D, Yang D, Zheng S, Hao K, Bosse Y, et al. Identification of TMPRSS2 as a susceptibility gene for severe 2009 pandemic $A(H 1 N 1)$ influenza and $A(H 7 N 9)$ influenza. J Infect Dis. 2015;212(8):1214-21.

11. Balcarek J, Sevá Pessôa B, Bryson C, Azizi M, Ménard J, Garrelds IM, McGeehan G, Reeves RA, Griffith SG, Danser AHJ, et al. Multiple ascending dose study with the new renin inhibitor VTP-27999: nephrocentric consequences of too much renin inhibition. Hypertension. 2014;63(5):942-50.

12. Danser AHJ, Batenburg WW, van den Meiracker AH, Danilov SM. ACE phenotyping as a first step toward personalized medicine for ACE inhibitors. Why does ACE genotyping not predict the therapeutic efficacy of ACE inhibition? Pharmacol Ther. 2007;113(3):607-18.

13. Wei L, Alhenc-Gelas F, Soubrier F, Michaud A, Corvol P, Clauser E. Expression and characterization of recombinant human angiotensin $\mathrm{l}$-converting enzyme. Evidence for a $\mathrm{C}$-terminal transmembrane anchor and for a proteolytic processing of the secreted recombinant and plasma enzymes. J Biol Chem. 1991;266(9):5540-6.

14. Arendse LB, Danser AHJ, Poglitsch M, Touyz RM, Burnett JC Jr, Llorens-Cortes C, Ehlers MR, Sturrock ED. Novel therapeutic approaches targeting the renin-angiotensin system and associated peptides in hypertension and heart failure. Pharmacol Rev. 2019;71(4):539-70.

15. Wysocki J, Ye M, Khattab AM, Fogo A, Martin A, David NV, Kanwar Y, Osborn M, Batlle D. Angiotensinconverting enzyme 2 amplification limited to the circulation does not protect mice from development of diabetic nephropathy. Kidney Int. 2017;91(6):1336-46.

16. Serfozo P, Wysocki J, Gulua G, Schulze A, Ye M, Liu P, Jin J, Bader M, Myohanen T, Garcia-Horsman $\mathrm{JA}$, et al: Ang II (angiotensin II) conversion to angiotensin-(1-7) in the circulation is POP (prolyloligopeptidase)-dependent and ACE2 (angiotensin-converting enzyme 2)-independent. Hypertension 2020, 75(1):173-182.

17. Liu Y, Yang Y, Zhang C, Huang F, Wang F, Yuan J, Wang Z, Li J, Li J, Feng C, et al. Clinical and biochemical indexes from 2019-nCoV infected patients linked to viral loads and lung injury. Sci China 
Life Sci. 2020;63(3):364-74.

18. Rieder M, Wirth L, Pollmeier L, Jeserich M, Goller I, Baldus N, Schmid B, Busch HJ, Hofmann M, Kern W, et al. Serum ACE-2, angiotensin II, and aldosterone levels are unchanged in patients with COVID19. Am J Hypertens. 2020;34:278-81.

19. Henry BM, Benoit S, Lippi G, Benoit J. Letter to the Editor - Circulating plasma levels of angiotensin II and aldosterone in patients with coronavirus disease 2019 (COVID-19): A preliminary report. Prog Cardiovasc Dis. 2020;63(5):702-3.

20. Nussberger J, Brunner DB, Waeber B, Brunner HR. True versus immunoreactive angiotensin II in human plasma. Hypertension. 1985;7(3 Pt 2):11-7.

21. Campbell DJ, Kladis A, Skinner SL, Whitworth JA. Characterization of angiotensin peptides in plasma of anephric man. J Hypertens. 1991;9(3):265-74.

22. Chappell MC, Pirro NT, South AM, Gwathmey TM. Concerns on the specificity of commercial ELISAs for the measurement of angiotensin-(1-7) and angiotensin II in human plasma. Hypertension. 2021;77:e29-31.

23. Kintscher U, Slagman A, Domenig O, Rohle R, Konietschke F, Poglitsch M, Mockel M. Plasma angiotensin peptide profiling and ACE (angiotensin-converting enzyme)-2 activity in COVID-19 patients treated with pharmacological blockers of the renin-angiotensin system. Hypertension. 2020;76(5):e34-6.

24. Kutz A, Conen A, Gregoriano C, Haubitz S, Koch D, Domenig O, Bernasconi L, Mueller B, Schuetz P. Renin-angiotensin-aldosterone system peptide profiles in patients with COVID-19. Eur J Endocrinol. 2021;184(4):543-52.

25. Fang L, Karakiulakis G, Roth M. Are patients with hypertension and diabetes mellitus at increased risk for Covid-19 infection? Lancet Respir Med. 2020;8:e21.

26. Mancia G, Rea F, Ludergnani M, Apolone G, Corrao G. Renin-angiotensin-aldosterone system blockers and the risk of Covid-19. N Engl J Med. 2020;382(25):2431-40.

27. de Abajo FJ, Rodriguez-Martin S, Lerma V, Mejia-Abril G, Aguilar M, Garcia-Luque A, Laredo L, Laosa $\mathrm{O}$, Centeno-Soto GA, Angeles Galvez M, et al. Use of renin-angiotensin-aldosterone system inhibitors and risk of COVID-19 requiring admission to hospital: a case-population study. Lancet. 2020;395(10238):1705-14.

28. Wu C, Ye D, Mullick AE, Li Z, Danser AHJ, Daugherty A, Lu HS. Effects of renin-angiotensin inhibition on ACE2 (angiotensin-converting enzyme 2) and TMPRSS2 (transmembrane protease serine 2) expression: insights into COVID-19. Hypertension. 2020;76(4):e29-30.

29. Semenzato L, Botton J, Drouin J, Baricault B, Vabre C, Cuenot F, Penso L, Herlemont P, Sbidian E, Weill A, et al. Antihypertensive drugs and COVID-19 risk: a cohort study of 2 million hypertensive patients. Hypertension. 2021;77(3):833-42.

30. Kai H, Kai M, Niiyama H, Okina N, Sasaki M, Maeda T, Katoh A: Overexpression of angiotensinconverting enzyme 2 by renin-angiotensin system inhibitors. Truth or myth? A systematic review of animal studies. Hypertens Res 2021:in press. 
31. Jansen PM, van den Born BJ, Frenkel WJ, de Bruijne EL, Deinum J, Kerstens MN, Smulders YM, Woittiez AJ, Wijbenga JA, Zietse R, et al. Test characteristics of the aldosterone-to-renin ratio as a screening test for primary aldosteronism. J Hypertens. 2014;32(1):115-26.

\section{Figures}

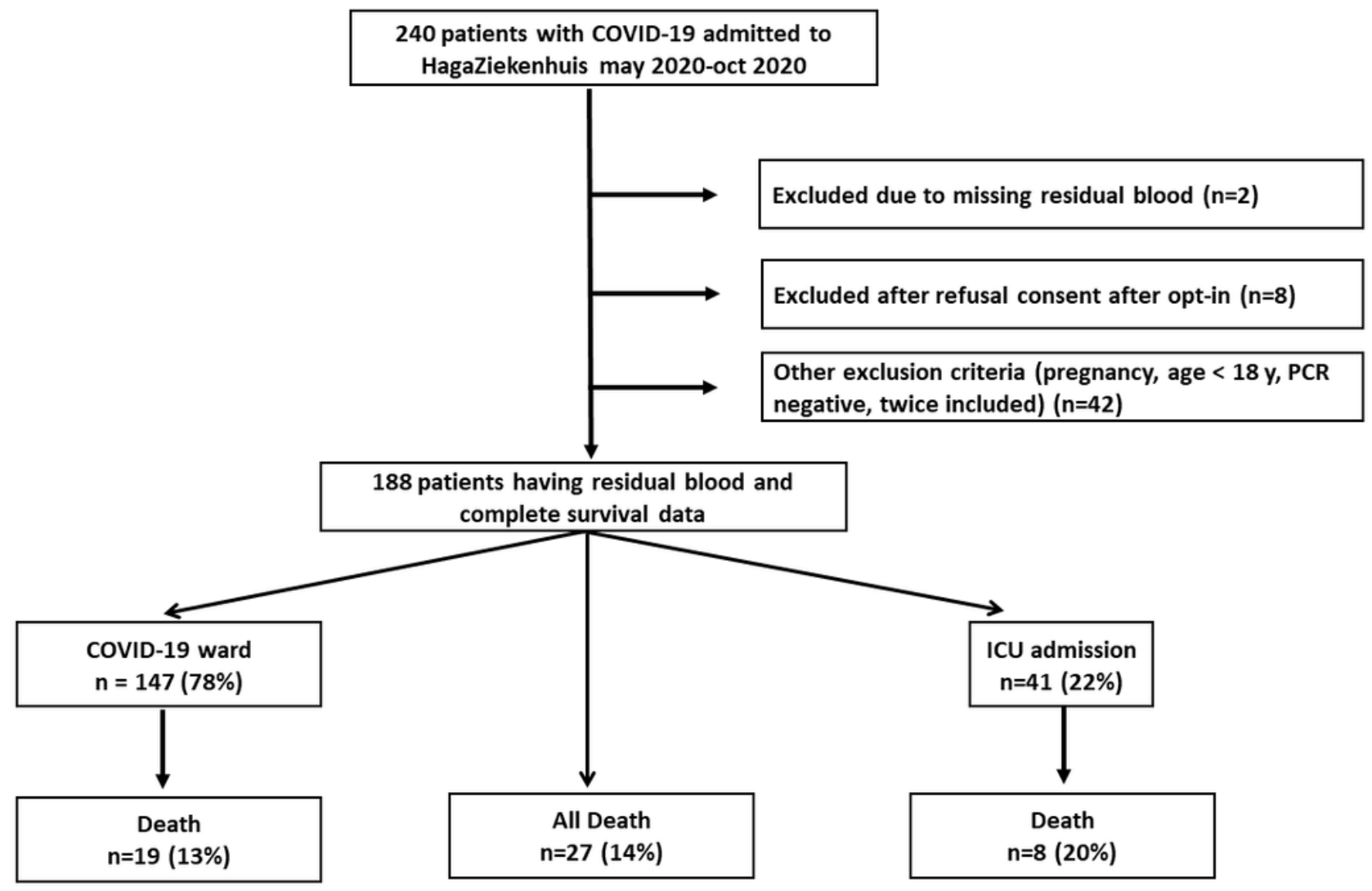

\section{Figure 1}

Of the 240 adult COVID-19 patients included in our database of whom residual blood samples were available between May 2020 and October 2020, 52 were excluded because of missing residual blood $(n=2)$, rejection of previous inclusion by opt-out consent $(n=8)$, or other exclusion criteria such as pregnancy, negative PCR and transportation to another hospital $(n=42)$ 
ACEi/ARB -

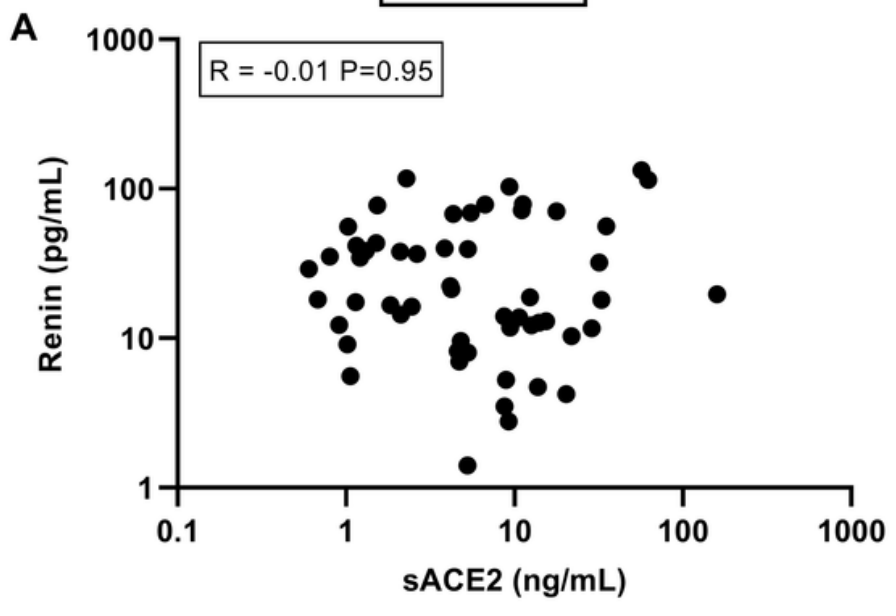

ACEi/ARB +

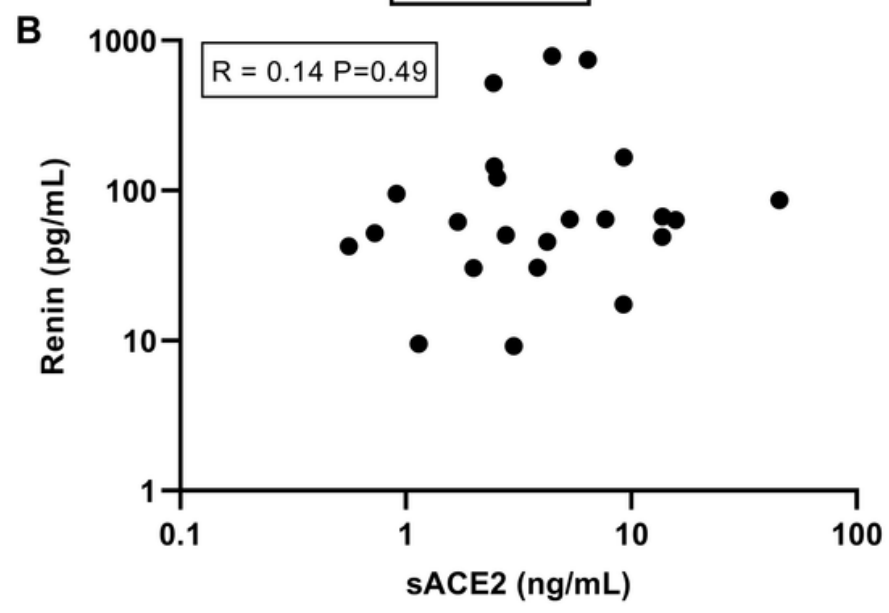

Figure 2

Renin did not correlate with SACE2, neither in subjects taking RAAS inhibitors, nor in subjects not taking these drugs
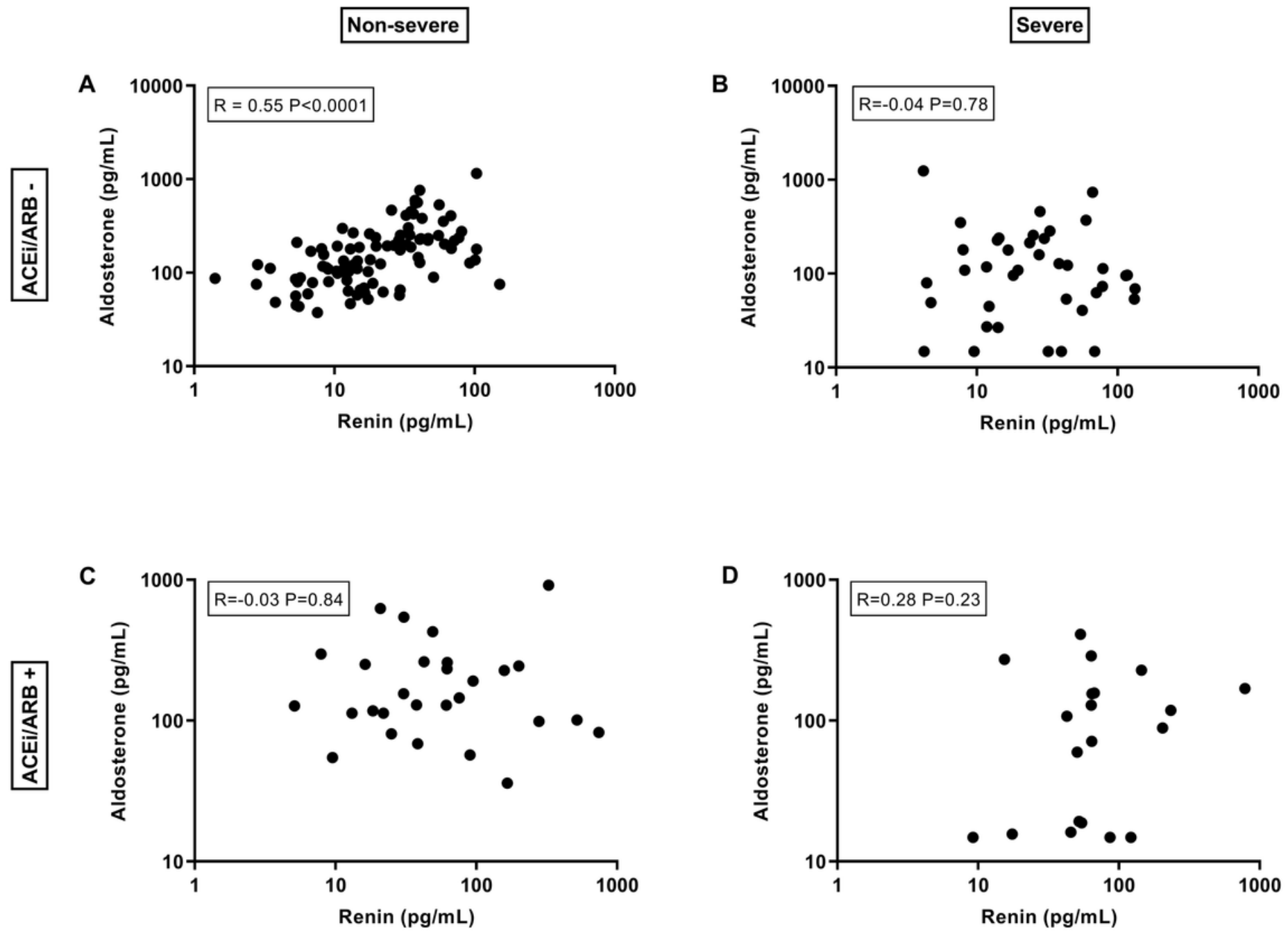
Figure 3

As expected, in non-severe COVID-19 patients renin correlated with aldosterone in those not taking RAAS inhibitors, but not in those taking such drugs 